\title{
Enhancing Detection Performance of Face Recognition Algorithm Using PCA-Faster R-CNN
}

\author{
Hashiru Isiaka Muhammad, Kabir Ibrahim Musa, Mustapha Lawal Abdulrahman, Abdullahi Abubakar, \\ Kabiru Umar, and Abdulhakeem Ishola
}

\begin{abstract}
In this paper, we present a new face detection scheme using deep learning and achieving state-of-the-art recognition performance using real-world datasets. We designed and implemented a face recognition system using Principal Component Analysis (PCA) and Faster R Convolutional Neural Network (Faster R CNN). In particular, we improve the state-of-the-art Faster RCNN framework by using Principal Component Analysis (PCA) technique and Faster $\mathrm{R} C N N$ to detect and recognise faces in a face database. The Principal Component Analysis (PCA) was used to extract features and dimensionality reduction from the face database, while the Faster $R$ Convolutional Neural Network algorithm was used to identify patterns in the dataset via training the neural network. The three real-world datasets used in our experiment are ORL, Yale, and California face dataset. When implemented on the ORL face dataset, the algorithm achieved average recognition accuracy of $99 \%$, with a recognition time of 147.72 seconds for 10 runs, and the recognition time/image was 0.3 sec/image on 400 images. The Yale face dataset achieved average recognition accuracy of $99.24 \%$ with a recognition time of 63.45 seconds for 10 runs, and the recognition time/image was 0.53 sec/image on 120 images. Finally, on California Face Database (CFD), it achieved average recognition accuracy of 99.52\% with a recognition time of 226.05 seconds for 10 runs, and the recognition time/image was 0.27 sec/image on 827 images. On the CFD dataset, however, the proposed approach has excellent classification performance when the recall ratio is high. The proposed method achieves a higher recall and accuracy ratio than the Faster RCNN without PCA method. For the F-score, the proposed method achieved 0.98 , which is significantly higher than the 0.95 achieved by the Faster-RCNN. This demonstrates the superiority of our model performancewise as against state-of-the-art, both in terms of accuracy and fast recognition. Therefore our model is more efficient when compared to the latest researches done in the area of facial recognition.
\end{abstract}

Index Terms - Face Recognition, Convolutional Neural Network (CNN), Faster R-CNN, Principal Component Analysis (PCA), PCA-Faster R-CNN.

\section{INTRODUCTION}

Face detection is the preliminary stage for all systems that interact with human robots, computer-based, and vision-

Submitted on April 20, 2021

Published on May 11, 2021.

Hashiru Isiaka Muhammad, Department of Mathematical Sciences, Abubakar Tafawa Balewa University, Bauchi, Nigeria.

(corresponding e-mail: hashiruisiaka@gmail.com)

Kabir Ibrahim Musa, Department of Mathematical Sciences, Abubakar Tafawa Balewa University, Bauchi, Nigeria.

(e-mail: imkabir@atbu.edu.ng)

Mustapha Lawal Abdulrahman, Department of Mathematical Sciences, Abubakar Tafawa Balewa University, Bauchi, Nigeria.

(e-mail: musbaida@gmail.com) based such as the ASIMO robot by Honda, which has advanced face detection and recognition component [3]. Face detection helps in tagging images on social media such as Facebook and Instagram, among others. Automatic face detection can be seen as the foundation stone of programs spinning around automatic facial images analysis, including face detection, face verification, gender or age recognition, face surveillance and tracking, relighting, and morphing. Embedded face detector can also be found in digital cameras and smartphones used to focus the image to be detected automatically [3].

Research has shown that automatic face detection and facial feature recognition were first computer vision-based applications [20]. Face recognition is a well-studied research area in computer vision. Recently build face detectors, such as "Face detection based on YOLOv3" [23], can easily detect frontal faces quickly and accurately on face detections task.

Most recent research areas concentrate mainly on uncontrolled facial recognition problems, where a variety of characteristics are considered; such as posing shifts, trilling lights, and exaggerated gestures. This can contribute to significant visual differences in the face's appearance and seriously weaken the facial detector's strength.

The challenges in identifying the face are primarily due to two aspects: (i) the large optical differences of human faces in a disorderly manner and (ii) the large area of search for potential characteristics and sizes of the face. The former allows the face detector to fix binary classification, while the latter sets a time efficiency criterion.

Viola \& Jones [35], proved that the improved cascade with simple qualities popularly becomes more effectively designed for practical face detection [38]. One of Viola and Jone's work's significant potential is its ability for accurate evaluation and quickly rejects false positive detections. The simple nature of the improved cascade qualities was constructed and assembled to achieve accurate face versus no-face sorting. The Haar mechanism was used to test the faces, and for frontal faces, it is found to be less biased. Due to the Hair function's simple design, the uncontrolled environment under which faces are in different places and expressions under unpredictable lighting is comparatively

Abdullahi Abubakar, Department of Computer Science, Waziri Umaru Federal Polytechnic, Birnin Kebbi, Kebbi State, Nigeria. (corresponding e-mail: abafo22@gmail.com)

Kabiru Umar, Department of Mathematical Sciences, Abubakar Tafawa Balewa University, Bauchi, Nigeria.

(e-mail: kumar@atbu.edu.ng)

Abdulhakeem Ishola, Department of Computer Science, Waziri Umaru Federal Polytechnic, Birnin Kebbi, Kebbi State, Nigeria. (e-mail: abduljedo@gmail.com) 
bad. Therefore, after the work done by Viola \& Jones [35], much research and enhancement have been achieved over the decades [32]. Most of them with more advanced features adopt the boosted cascade structure. At the cost of additional computation, the advanced function helps create a more accurate binary classifier. However, it is possible to reduce the number of cascade stages needed to achieve comparable detection precision. Therefore, due to fewer cascade phases, the overall estimate will remain the same or even decreased.

Because of its potential for multiple applications, Face recognition has attracted much interest from the research community: biometric identification software, it is used to search for a person through cameras, automatically tag friends on social media sites, and to find similar or identical individuals on a database either online or offline.

As a recent and exciting field of study which has been extended to various fields of facial detection and facial recognition, raw data can be mapped from multiple to Euclidean space in deep convolutional neural network (CNN) in which features can be linearly separated. Mostly, for deep CNN models, there are two ways to derive functions. The first approach is by extracting the image cascade features obtained using methods of generating object proposals such as Selective Search [33] Edge boxes [39], and Cohesion measurement CM [12]. However, this method has an enormous computational challenge. The second approach is by using the sliding window technique on convolutional feature maps to obtain features such as R-CNN [10] and DenseNet [17].

When the sliding window moves at each location, the second approach is generally more effective in obtaining features than the first approach. One of the challenges second approach's is the obtained features are less known than those obtained by the first approach. However, for object detection, there is high computational complexity in both approaches. Selective search is used by both of the algorithms above i.e. R-CNN and Fast R-CNN.

Selective search is a time-consuming and inefficient process that impacts network efficiency [10]. As the newest generation of generic object detection methods based on region, the Faster R-CNN demonstrates promising results on different benchmarks for object detection. A function representation from data can be learned automatically by Faster R-CNN [18]. The Faster R-CNN enables all levels of end-to-end learning, enhancing its robustness relative to other neural methods [18]. The Faster R-CNN model has demonstrated that it can learn to cope with occlusions with lower computational time using the Faster R-CNN for face detection [13]. Therefore, because of the existing object identification criterion, there is a need to enhance the detection efficiency, which remains a very important unsolved issue in the field of computer vision. Based on the literature, Principal Component Analysis (PCA) has been proven to increase the algorithm's efficiency. Li et al [22], has shown that the classification algorithm's performance is enhanced by reducing the dimensionality without losing information from any features. Additionally, PCA reduces the storage space used to store data and speeds up the learning algorithm due to a lower dimension. Furthermore, PCA fixes the problem of multicollinearity (all major components are orthogonal to each other) and help visualise highdimensionality data (after reducing the dimension) [22].

Using Faster R-CNN, the existing system attempts to detect faces. The use of only several convolution layers for feature extraction from the data set is one of the disadvantages of the existing system. The current approach uses CNN, which usually is very effective as the sliding window changes at each location to obtain characteristics. However, one of the challenges with this approach is that the computational complexity is high for object detection compared to the recent Faster-CNN. Selective search is used by each of the above-listed algorithms (R-CNN \& Fast R$\mathrm{CNN}$ ) to figure out the field proposals. Selective search is a slow and time-consuming process that reduces the network's performance [28]. R-CNN needs a significant amount of time to train the network. It would be difficult to locate 2000 regional proposals per picture in real-time, taking around 47 seconds for each test image to be implemented. A fixed algorithm is the Restricted Search Algorithm. Therefore, at that point, no learning is occurring. This could lead to bad applicant area proposals being produced.

Therefore, this work employs the use of Principal Component Analysis (PCA) to extract the feature and feed it to Faster-multiple R-CNN's convolution layers for further processing to improve the face recognition model's efficiency. Our proposed model is designed for faster and accurate face detection. We used standard metrics to evaluate our model's performance, such as recognition accuracy, accuracy precision, recall, and F1-Measure. Our model shows a better result compared to state of the art.

\section{A. Contributions}

Compared to other states of the art models, this research increased the accuracy and reduced the computational complexity significantly. We improve the state-of-the-art Faster RCNN framework by using Principal Component Analysis (PCA) technique and Faster R CNN to detect and recognise faces in a face database. The Principal Component analysis (PCA) was used to extract features and dimension reduction from the face database and Faster $\mathrm{R}$ Convolutional Neural Network algorithm was used to identify patterns in the dataset via training the neural network.

The remainder of this paper is organised as follows. We discussed the related work in Section II; this includes the theoretical history of Deep Learning approaches incorporated into the proposed models. Section III; presents the formulation and overview of the proposed model and details how the model was implemented. Section IV; presents the results, demonstrating the capability of our method. Finally, we provide the summary, conclusion and routes for possible future work in Section V.

\section{RELATED WORK}

This Section reviews current and relevant literature with extensive analysis of various theoretical frameworks and methodologies used for face detection modeling. An outline of the diverse approaches used was critically analysed concerning strengths, drawbacks, and gaps in previous research works. 


\section{A. Relevant Work}

The success of the Viola-Jones detector was based on two factors: the rapid assessment of the features of the Haar and the cascaded structure that enables early pruning of false positives [15]. Recent work done by [31], demonstrate better face detection performance using the Improved Viola-Jones Algorithm. The multi-threaded cascade of Speed Up Robust Features (McSURF) was used for facial expression recognition. The precision of the $\mathrm{CK}+$ database was $87.5 \%$. [1], used Local Binary Patterns Histogram (LBPH) Based Face Recognition At Low Resolution to obtain a recognition accuracy of 90\%. [16], applied Local Binary Pattern Algorithm for face recognition application with high accuracy.

Liqiao \& Runhe [25], used the algorithm on adaptive weighted Centered Gradient Histograms (HOG) to detect face with $76 \%$ accuracy. The Fisher Linear Discriminant Analysis algorithm is also used [27] to classify faces with different expressions and to recognise the face with attribute changes such as glasses, whiskers, and others with $90 \%$ precision. For makeup-invariant face recognition and verification, Kamil \& Are [19] proposed a hybrid feature extraction technique [19]. For feature extraction, the Gabor Filter Bank (GFB) and Histogram of Directed Gradients (HOG) were applied to face images from the Virtual Makeup (VMU) database. Using a combination of GFB and HOG characteristics, the final feature vectors were generated and categorised using City Block Distance (CBD), Euclidean Distance (EUC) and Cosine Similarity Metrics (CSM). For the VMU database, the performance assessment of the recognition and authentication rates supplied by the CBD, EUC, and CSM classifiers was between $89.54 \%$ and $92 \%$.

Yang \& Yang [37], reported that, compared to holistic and local methods, the hybrid methods used in face recognition problems have a high accuracy rate. Sun \& Yu [30] used the dual-tree dynamic wavelet transformation and gray level cooccurrence matrix. While Allagwail [2] merged Local Binary Patterns and Gabor Filter with high precision for use with facial recognition.

Over a decade, researchers have developed different types of facial recognition algorithms. These include the Sparse Coding (SC), the Local Binary Pattern, algorithm, (LBP) algorithms, the Directed Gradient Histograms (HOG) algorithm, the Linear Discriminant Analysis (LDA) algorithm, and the Gabor algorithm. Many of these algorithms have an accuracy rating of between $50 \%$ and $76 \%$.

Chen et al. [5], used Supervised Transformer Network with enhanced precision for face detection using. CNN for face recognition using Georgia Tech Database. Coskun et al. [7], shows that the proposed methodology has improved face detection efficiency with greater identification outcomes than traditional approaches. In order to illustrate a model trained using multiple Facial Recognition datasets, CNN visualisation tools were used. Authors in [4] demonstrated the potential of emotion detection-trained networks in both datasets and various FER-related activities. A Convolutional Neural Network (CNN) Method for Face Detection and Recognition has been adopted (DEFFO, Tonye, \& FUTE) using a limited data set in Cameroon with an accuracy of below $91 \%$. There has long been a history of training neural networks for face recognition. Although $\mathrm{CNN}$ has demonstrated good object detection performance, its computational efficiency is not high.

Deeply trained neural networks, most prominently CNN's, have revolutionised a variety of computer vision tasks, including face recognition, according to Jiang \& LearnedMiller [18], as witnessed by the increasing success of the Face Detection Database and Benchmark (FDDB). In comparison with non-neural methods, the Faster R-CNN will automatically learn a feature representation from data, which typically focus on hand-crafted characteristics. The Faster RCNN enables all levels of end-to-end learning, enhancing its robustness relative to other neural methods. [18]

Using the Faster R-CNN for face detection was studied [13], which revealed that the Faster R-CNN model would learn to cope with occlusions only from data with less computational time. According to Guo et al. [11], the identification of Over Feat takes a long time since the classifier, and regressor networks must be run through all possible threshold settings. R-CNN produces multiple classindependent recommendation windows first and then extracts features from $\mathrm{CNN}$ model windows trained on a multi-scale picture pyramid to maximise performance [11].

Therefore, the face recognition system is proposed in this study using the hybrid Principal Component Analysis (PCA) and Faster R CNN process.

\section{B. Overview of Computational Algorithms}

\section{Convolutional Neural Network (CNN)}

A convolutional neural network (CNN or ConvNet) is a type of deep neural network that is most frequently used in deep learning to explore visual images [34]. Many computer visions functions have been controlled by deep convolutional neural networks (CNNs). The dominant model is now the region-based convolutional neural network (R-CNN) detection methods for target detection. R-CNN is briefly discussed below.

\section{2. $R-C N N$}

The Region-based CNN detector [10] generates field proposals using an algorithm such as Edge Boxes [39]. The proposed regions are taken out of the image and resized. CNN would then categorise the cropped and resized regions. Finally, the bounding boxes of the field proposal are refined by a support vector machine (SVM) trained with $\mathrm{CNN}$ features.

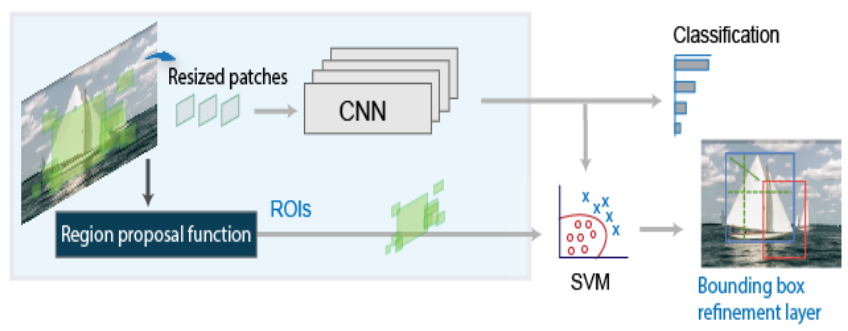

Fig. 1. The Region-based CNN detector [10] \& [28].

According to Girshick et al. [10], the bounding-box image processing and analysis approach of the Region-based CNN (R-CNN), is to consider the implications of a manageable number of candidate object regions. Uijlings et al. [33], Girshick [9] and independently test coevolutionary networks [21] on each RoI. R-CNN. The study of [14] and [9] has been 
expanded to allow RoIPool to participate in ROIs on feature maps, resulting in faster speed and better accuracy.

\section{Faster $R-C N N$}

A region proposal network (RPN) is introduced by the Faster R-CNN [28] detector to produce region proposals directly on the network instead of using an external algorithm such as Edge Boxes. The RPN uses Anchor Boxes for Object Recognition. The development of area proposals in the network is faster and better suited to your results.

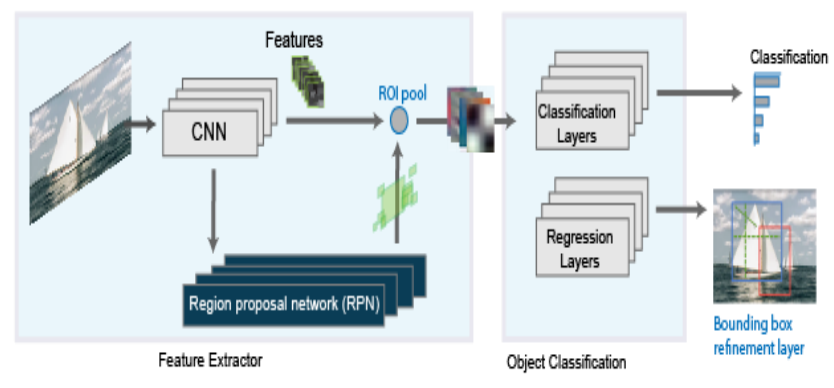

Fig. 2. Faster R-CNN [10]\&[28].

\section{PCA for Feature Extraction}

PCA is a common technique used for approximating lower-dimensional feature vectors to the original data. The facial expression of the PCA algorithm is used as a random vector. A group of new orthogonal bases is obtained by solving the scatter matrix eigenvalue problem of training samples to show the subspace spanned by training samples, and the characteristics extracted are only project-vectors of the face images [24].

The extraction of the PCA features is done by training the samples to obtain a subspace consisting of orthogonal base vectors, then projecting the samples into the subspace as the sample function vectors will obtain projection coefficient vectors. Likewise, the feature vectors of the test samples can be obtained by mapping the test sample pictures into the subspace. In this way, the topic of face recognition becomes an issue of projection coefficient vector classification. For face recognition, PCA may therefore be used [24].

\section{MethodOLOGY}

This study aims to use Principal Component Analysis (PCA) to extract features from the face dataset and then use Faster R-CNN to identify patterns in the dataset to build and deploy a face recognition model. Compared to other state-ofthe-art models, this research would increase the accuracy of recognition significantly.

In recent times, Hybrid Deep learning models have produced remarkable results for multiple machine vision applications. For the identification of the face, the derived characteristics are sparse and indifferent to size variations. As a result, the direct description of the extracted facial features greatly increases the performance of facial recognition models relative to other state-of-the-art face detection approaches using CNN, R CNN and Faster RCNN models.

The three key components of the proposed Hybrid PCAFaster R-CNN Fast-Face Recognition System are defined in such a way that we build a very minimal feature space added to the PCA and then feed the rectified linear unit (ReLU). Secondly, the multi-scale features have the same size as the size of the fully-connected layer. Third, the network is qualified to achieve the requisite precision. As a result, the complete linked layer in Faster $\mathrm{R} \mathrm{CNN}$ can be further calculated as a linear classification for a linear classifier for the model of face recognition.

The raw data set has excessive noise, large dimensionality, and very high variability, hence the need to use an additional feature extraction scheme such as the PCA technique. PCA of Bernstein[40], is used for feature extraction and dimension reduction in a data set. In addition, because of the real-time nature of object detection, it remains a difficult challenge to improve detection efficiency. To enhance the quality of the algorithm based on the literature, PCA has shown that the classification algorithm performance is improved by reducing the dimensionality without losing information from any features, reducing the storage space required to store data, speeding up the learning algorithm (with lower dimension). Fix the problem of multicollinearity (all key components are orthogonal to each other) and help visualise highdimensionality data (after reducing the dimension). Our proposed framework is shown in Fig. 3.

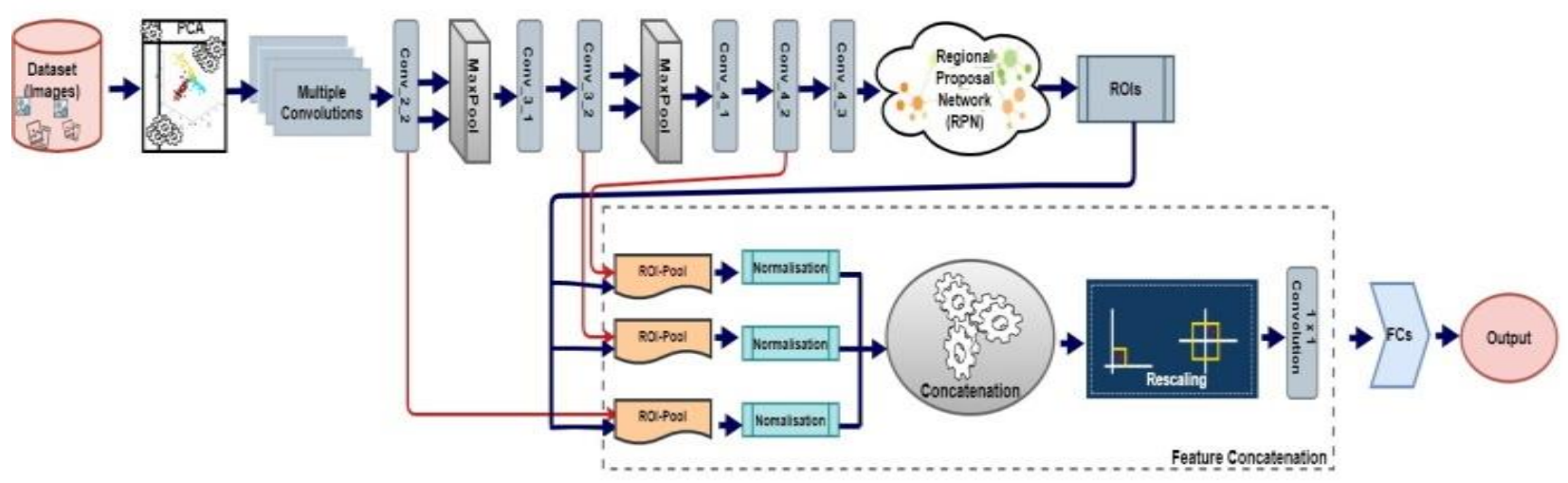

Fig. 3. Architecture of the proposed system.

\section{A. Model Formulation}

The facial data set is fed as an input to the PCA unit built on the top layer of the neural network of the Faster R-CNN. The PCA unit then performs features extraction and dimension reduction on the data set. Multiple convolution layers, Convolution layers, Max Pool Layers, RPN and Network, ROI pools, and completely linked layers make up the performance of the PCA unit as input to the Faster RCNN 
network. For pattern classification applications, the Faster $\mathrm{R}$ CNN network is a type of Neural Network (NN) that can learn dependencies between the data and extract patterns. This work adopts the Faster R-CNN model architecture to training the network and learns the relevant patterns to enhance the recognition accuracy inherent in the model.

\section{B. Hard Negative Mining}

Hard negative mining is a common approach in object detection, where training and testing procedures are alternated to recognise the hard negative, such as traditional false positives [26]. Hard negative mining is a useful technique for enhancing the efficacy of deep learning, particularly for object recognition tasks such as face detection [36]. The principle behind this approach is that, the regions where the network has failed to make the right prediction are harsh negatives. Thus, as a reinforcement to improve our qualified model, the hard negatives are being pumped back into the network. The subsequent training stage will then strengthen our model for fewer false positives and improved classification outcomes. From the first phase of our training process, the hard negatives are harvested from the pre-trained model. The hard negatives were added directly to the ROIs during the hard-negative training process for fine-tuning the model and balancing the foreground and background ratio to around 1:3, which is the same as the ratio we use in the first level.

\section{Data Collection (Dataset)}

The study aims to use a collection of facial images from the ORL Olivetti Research Labs (ORL), now AT\&T, Yale Face Database, and California Face Database for research purposes. The database was used in the sense of a face recognition project carried out in collaboration with the Cambridge University Engineering Department's Voice, Vision, and Robotics Division.

\begin{tabular}{cccc}
\multicolumn{4}{c}{ TABLE I: DATASET SUMMARY } \\
\hline \multirow{2}{*}{ Dataset } & Training & Testing Samples & Properties \\
& Sample & 120 & grayscale \\
& 400 & 50 & grayscale \\
ORL & 165 & 248 & colored \\
Yale & 827 & & \\
\hline
\end{tabular}

\section{System Specification}

The work is implemented using MATLAB version 2019a on HP laptop System CoreTMi7, 1.7GHz CPU and 8GB RAM. The performance of the proposed algorithm was implemented using benchmark face database of Olivetti Research Labs (ORL) now known as AT\&T, California Face Database (CFD) and Yale face database. Table II shows the summary of the dataset.

\section{E. Evaluation Parameters}

In this study, the model performance evaluation parameter is the computational time and recognition accuracy which is measured in percentages. When the proposed model has achieved the desired objective, which is feature selection and classification, the model will be evaluated based on the performance obtained. This work makes use of accuracy as the performance metrics.
Accuracy: This performance metric deal with the correct prediction made by the model and this metric can be expressed as:

$$
\text { Accuracy }=\frac{\mathrm{TP}+\mathrm{TN}}{\mathrm{TP}+\mathrm{FP}+\mathrm{FN}+\mathrm{TN}}
$$

where TP (True Positive) = are the cases where the actual class of the data point was 1 (true) and the prediction is also 1 (true).

TN $($ True Negative $)=$ These are the cases where 0 (false) was the real data point class and 0 was also the expected data point class (false).

FP $($ False Positive $)=$ These are the cases in which 0 (false) was the real data point class and 1 is the expected value (true). FN $($ False Negative $)=$ These are cases in which 1 (true) is the real data point class and 0 is the expected data point class (false).

\section{RESUlts PRESENTATION AND ANALYSIS}

A detailed feasibility review of the proposed face detection scheme is provided in this section. Using two public datasets, we present efficiency and output analysis between the solution suggested and state-of-the-art face detection methods.

\section{A. The Performance Comparison for Face Recognition with Other Studies}

Discrete firefly Algorithm (DFA), Firefly Algorithm (FA), Particle Swarm Optimization (PSO), Genetic Algorithm (GA), and Binary Particle Swarm Optimization (BPSO) Algorithm were used to compare the proposed algorithm to other meta-heuristic algorithms developed by other researchers. Table II summarises the results of the comparison of the proposed PCA-FR-CNN for feature selection in face recognition with the other algorithms using the ORL Face Database. On the ORL dataset, the proposed algorithm has a higher recognition accuracy than previous Face Recognition works on the same dataset. This is because the proposed model's Faster R-CNN is a special case of the SPPnet, which uses a single spatial pyramid pooling layer, i.e., the ROI pooling layer, and thus allows end-to-end finetuning of a pretrained ImageNet model. This is why it performs better than other face recognition approaches used in this study [13].

TABLE II: COMPARISON OF PROPOSED METHOD WITH OTHER ALGORITHMS USING ORL FACE DATABASE

\begin{tabular}{cccc}
\hline Approach & Classifier Used & $\begin{array}{c}\text { Recognition } \\
\text { Accuracy }(\%)\end{array}$ & $\begin{array}{c}\text { Images } \\
\text { Used }\end{array}$ \\
\hline GA & K-NN & 90.50 & 5 \\
BPSO & Nearest Neighbor & 93.25 & 5 \\
PSO & MLP & 90.00 & 5 \\
FA & Nearest Neighbor & 94.38 & 5 \\
DFA & Nearest Neighbor & 97.75 & 5 \\
Faster R-CNN & CoV+Pool & 98.02 & 5 \\
Proposed & SoftMax Pixel & 99.00 & 5 \\
Model & & & \\
\hline
\end{tabular}

The above comparison for the recognition accuracy in Table II is presented graphically in Fig. 4 to have a better understanding of the trend. 
When implemented on the ORL face database, our algorithm achieved average recognition accuracy of $99 \%$, with a recognition time of 147.72 seconds for ten runs. This implies the recognition time per image was 0.3 sec/image on 400 images used. Fig. 5 is the comparison between our proposed algorithm and Faster- R-CNN on ORL dataset.

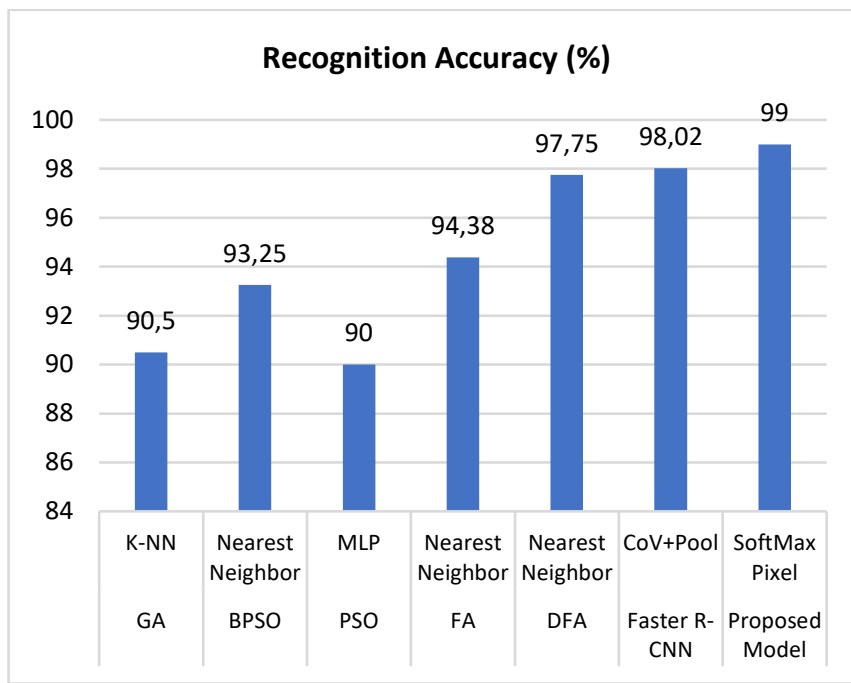

Fig. 4. Comparison of recognition accuracy against existing algorithm on ORL datasets.

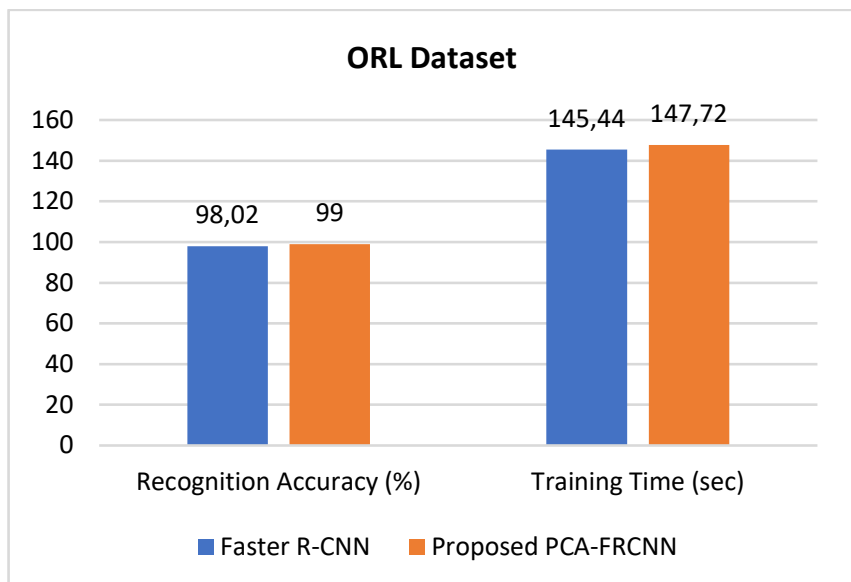

Fig. 5. Performance Comparison between proposed algorithm and Faster-RCNN on ORL datasets.

For ten runs on the Yale face database, the proposed algorithm achieved an average recognition (detection) accuracy of $99.24 \%$ and a recognition time of 63.45 seconds. This means that each image took 0.53 seconds to recognise. Fig. 6 is the comparison between our proposed algorithm and Faster- R-CNN on Yale dataset.

Finally, ten runs on the California Face Database (CFD) revealed that the proposed algorithm had an average recognition accuracy of $99.52 \%$ and a recognition time of 226.05 seconds. Once again, the picture recognition time was 0.27 seconds.

Hence, the larger the dataset, we observed a slight increase in accuracy. This was probably due to the large data sample required by deep learners to train well for an accurate classification model.

Again, Fig. 7 is the comparison between our proposed algorithm and Faster- R-CNN on CFD dataset.

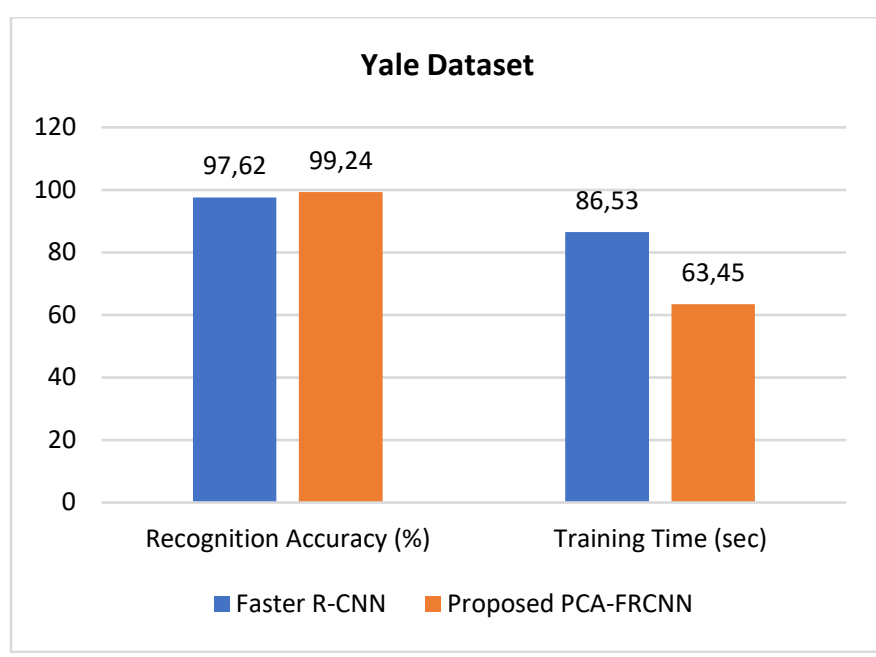

Fig. 6. Performance Comparison between proposed algorithm and Faster-R$\mathrm{CNN}$ on Yale datasets.

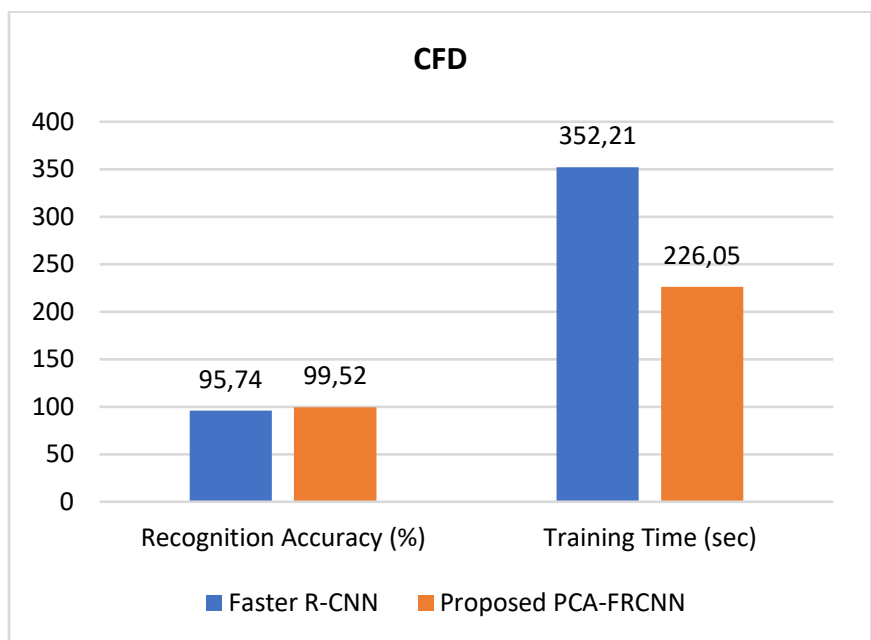

Fig. 7. Performance Comparison between proposed algorithm and Faster-RCNN on CFD datasets.

\section{B. Performance Comparison between Faster-RCNN and Fast-RCNN}

The computational complexity of our proposed method and the Fast-RCNN method for face detection is compared in this section. Table III, shows the comparison of the performance of the proposed simulated methods for face recognition to all other methods. We report the Precision, Recall and F-Score measure to evaluate the proposed PCAFRCNN on all the datasets. Table III depicts the result obtained after the simulation.

TABLE III: COMPARISON OF PROPOSED METHOD USING VARIOUS DATASETS WITH THE DIFFERENT NUMBER OF IMAGES

\begin{tabular}{ccccc} 
Method & Dataset & Precision & Recall & F-Score \\
\hline $\begin{array}{c}\text { Proposed PCA- } \\
\text { FRCNN }\end{array}$ & ORL & 0.97 & 0.93 & 0.98 \\
$\begin{array}{c}\text { Proposed PCA- } \\
\text { FRCNN } \\
\begin{array}{c}\text { Proposed PCA- } \\
\text { FRCNN }\end{array}\end{array}$ & Yale & 0.98 & 0.95 & 0.99 \\
\hline
\end{tabular}

The summary of the comparison between our proposed algorithm and Faster- R-CNN on all the datasets in Table III is represented graphically in Fig. 8. 


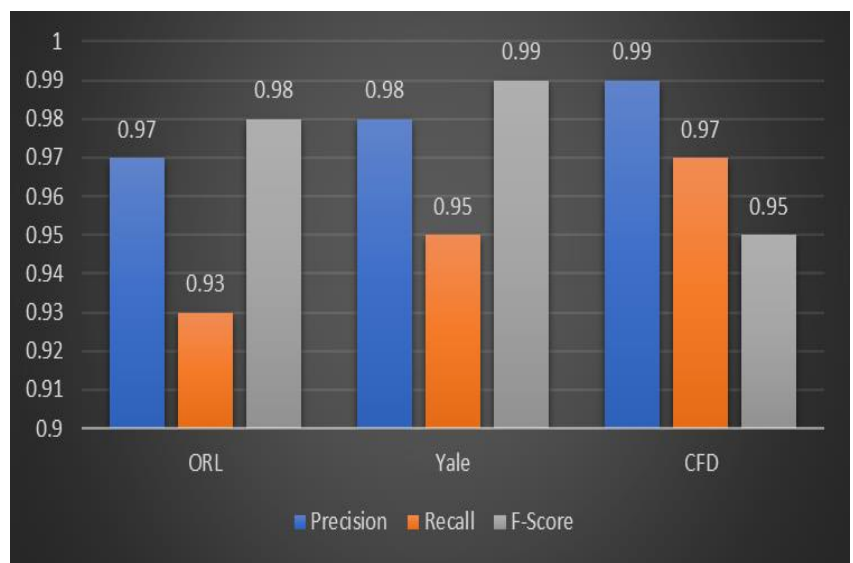

Fig. 8. Precision, Recall and F-Score measure comparison of our proposed model on all the three datasets.

On the ORL Face Database, the proposed method achieves an average precision of 0.97 , which is very fine. On the CFD dataset, however, the proposed approach has excellent classification performance when the recall ratio is high.

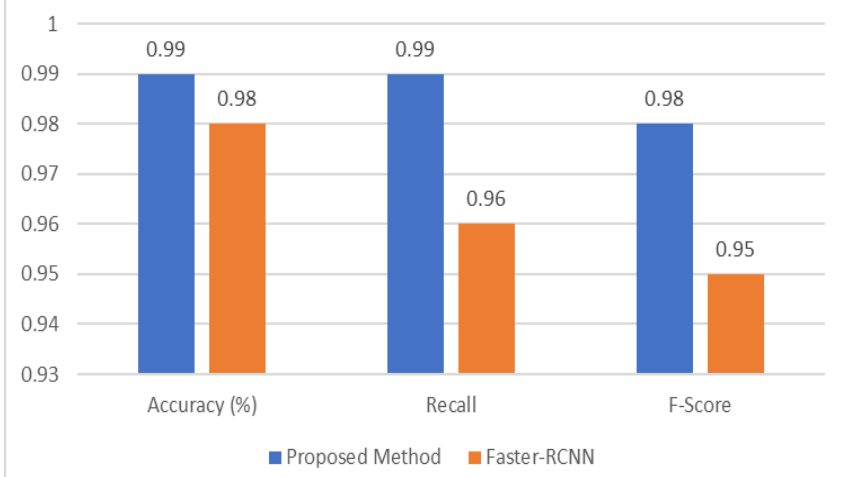

Fig. 9. Performance Comparison between proposed algorithm and Faster-R-CNN in term Recall and Accuracy.

Fig. 9 clearly shows that the accuracy, recall and $F_{1}$ score obtained by the proposed model was higher than the state-ofthe-art (the Faster RCNN). For instance, we attained $0.98\left(\mathrm{~F}_{1}\right.$ score), which is significantly higher than the 0.95 achieved by the Faster-RCNN. This demonstrates the superiority of the proposed model performance-wise against the state-of-theart.

We also compare the computational complexity. We use images with a resolution of $800 \times 600$ pixels for evaluation and presume five scales are used for detection. On the $800 \times 600$ picture, Table IV shows how much CPU time the proposed Faster R-CNN based face detection methods use.

TABLE IV: THE CPU TIME (IN SECONDS) USED By PROPOSED SYSTEM

\begin{tabular}{cccc}
\multicolumn{4}{c}{ AGAINST THE EXISTING SYSTEM ON ALL THE DATASETS } \\
\hline Method & ORL & YALE & CFD \\
\hline Proposed Model & 147.72 & 63.45 & 226.05 \\
Faster R-CNN & 145.44 & 86.53 & 352.21 \\
\hline
\end{tabular}

Table IV shows that the proposed method outperforms the current method in-terms of theoretical computational complexity (required FLOPS) and running time.

In addition, the proposed face detection approach is faster than the R-CNN methods. The explanation for this is that the current methods' CNN models are more complex than the proposed method's, requiring more computations.
Furthermore, the Faster-object RCNN's proposal generation methods consume more CPU time than the proposed process.

The above experiments show that the proposed technique can classify data with a high degree of dimensionality. It has the ability to recognise objects with a high degree of accuracy. Our proposed method outperforms the current state-of-the-art in terms of recognition accuracy and speed.

\section{CONCLUSION}

One of the major problems with the previous study on facial recognition is that the obtained features are less recognised, and the computational complexity is high for object detection. As the latest generation of region-based generic object detection approaches, Faster R-CNN displays promising performance on numerous object detection benchmarks. A function representation can be automatically learned from data by Faster R-CNN. As a result of the realtime requirement for object detection, it was found to be a very challenging issue on how to increase the effectiveness of the detection.

This study, therefore, suggested using Principal Component Analysis (PCA) and Faster R Convolutional Neural Network to implement a face recognition method. To extract features from the facial database we used the Principal Component Analysis (PCA). The Faster R Convolutional Neural Network algorithm was used to classify patterns in the dataset through neural network training.

The simulation findings suggest that the proposed solution is far more effective than the existing system. In our proposed method, the techniques used operate faster than using only the faster R-CNN methods. On the ORL Face Database, the proposed method achieves an average precision of 0.97 , which is very fine. On the CFD dataset, however, the proposed approach has excellent classification performance when the recall ratio is high.

The recall and accuracy ratio obtained by the proposed method was higher than the existing system. For the F-score, our proposed method achieved 0.98, which was also significantly higher than the 0.95 achieved by the FasterRCNN. This demonstrates the superiority of the proposed model performance-wise as against state-of-the-art, both in terms of accuracy and fast recognition.

\section{LIMITATIONS AND RECOMMENDATIONS}

One of the key drawbacks of this analysis is the availability of big data to ensure more excellent model reliability on broader datasets. Compared to other media, the amount of information necessary to train an efficient and robust deep learning model would be much greater. Training the model with larger data samples would ensure reliability and robustness.

Larger datasets should be used in future work. Additionally, we plan to expand our research to include other facial characteristics, such as tribal marks and similar features. 


\section{ACKNOWLEDGEMENT}

The Tertiary Education Trust Fund (TETFund) Institutional Based Research (IBR) Fund, through the Research and Innovation Directorate of Abubakar Tafawa Balewa University, Bauchi, funded this research.

\section{REFERENCES}

[1] Ahmed, A., Guo, J., Ali, F., Deeba, F., \& Ahmed, A. (2018). LBPH based improved face recognition at low resolution. Paper presented at the 2018 International Conference on Artificial Intelligence and Big Data (ICAIBD).

[2] Allagwail, S., Gedik, O. S., \& Rahebi, J. (2019). Face Recognition with Symmetrical Face Training Samples Based on Local Binary Patterns and the Gabor Filter. Symmetry, 11(2), 157.

[3] Sakagami, Y., Watanabe, R., Aoyama, C., Matsunaga, S., Higaki, N., \& Fujimura, K. (2002). The intelligent ASIMO: System overview and integration. In IEEE/RSJ international conference on intelligent robots and systems (Vol. 3, pp. 2478-2483). IEEE

[4] Breuer, R., \& Kimmel, R. (2017). A deep learning perspective on the origin of facial expressions. arXiv preprint arXiv:1705.01842.

[5] Chen, D., Hua, G., Wen, F., \& Sun, J. (2016). Supervised transformer network for efficient face detection. Paper presented at the European Conference on Computer Vision.

[6] Chen, J., Luo, Z., Takiguchi, T., \& Ariki, Y. (2016). Multithreading cascade of SURF for facial expression recognition. EURASIP Journal on Image and Video Processing, 2016(1), 37

[7] Coşkun, M., Uçar, A., Yildirim, Ö., \& Demir, Y. (2017). Face recognition based on convolutional neural network. Paper presented at the 2017 International Conference on Modern Electrical and Energy Systems (MEES).

[8] DEFFO, L. L. S., FUTE, E. T., \& Tonye, E. CNNSFR: A Convolutional Neural Network System for Face Detection and Recognition.

[9] Girshick, R. (2015). Fast $r$-CNN. Paper presented at the Proceedings of the IEEE international conference on computer vision.

[10] Girshick, R., Donahue, J., Darrell, T., \& Malik, J. (2014). Rich feature hierarchies for accurate object detection and semantic segmentation. Paper presented at the Proceedings of the IEEE conference on computer vision and pattern recognition.

[11] Guo, G., Wang, H., Yan, Y., Zheng, J., \& Li, B. (2019). A fast face detection method via a convolutional neural network. Neurocomputing.

[12] Guo, G., Wang, H., Zhao, W.-L., Yan, Y., \& Li, X. (2017). Object discovery via cohesion measurement. IEEE transactions on cybernetics, 48(3), 862-875.

[13] Hao, L., \& Jiang, F. (2018). A New Facial Detection Model based on the Faster R-CNN. Paper presented at the IOP Conference Series: Materials Science and Engineering.

[14] He, K., Zhang, X., Ren, S., \& Sun, J. (2015). Spatial pyramid pooling in deep convolutional networks for visual recognition. IEEE transactions on pattern analysis and machine intelligence, 37(9), 19041916.

[15] Huang, J., Shang, Y., \& Chen, H. (2019). Improved Viola-Jones face detection algorithm based on HoloLens. EURASIP Journal on Image and Video Processing, 2019(1), 41.

[16] Humne, S., \& Sorte, P. (2018). A Review on Face Recognition using Local Binary Pattern Algorithm.

[17] Iandola, F., Moskewicz, M., Karayev, S., Girshick, R., Darrell, T., \& Keutzer, K. (2014). Densenet: Implementing efficient convnet descriptor pyramids. arXiv preprint arXiv:1404.1869.

[18] Jiang, H., \& Learned-Miller, E. (2017). Face detection with the faster $R$-CNN. Paper presented at the 2017 12th IEEE International Conference on Automatic Face \& Gesture Recognition (FG 2017).

[19] Kamil, I. A., \& Are, A. S. (2018). Makeup-Invariant Face Recognition using combined Gabor Filter Bank and Histogram of Oriented Gradients. Paper presented at the Proceedings of the 2nd International Conference on Advances in Image Processing.
[20] Ko, B. (2018). A brief review of facial emotion recognition based on visual information. sensors, $18(2), 401$.

[21] Krizhevsky, A., Sutskever, I., \& Hinton, G. E. (2012). Imagenet classification with deep convolutional neural networks. Paper presented at the Advances in neural information processing systems.

[22] Li, C., Diao, Y., Ma, H., \& Li, Y. (2008). A statistical PCA method for face recognition. Paper presented at the 2008 Second international symposium on intelligent information technology application.

[23] Li, C., Wang, R., Li, J., \& Fei, L. (2020). Face detection based on YOLOv3. In Recent Trends in Intelligent Computing, Communication and Devices (pp. 277-284). Springer, Singapore.

[24] Li, J., Zhao, B., \& Zhang, H. (2009). Face recognition based on PCA and LDA combination feature extraction. Paper presented at the 2009 First International Conference on Information Science and Engineering.

[25] Liqiao, J., \& Runhe, Q. (2017). Face recognition based on adaptive weighted HOG. Computer Enigeering and Applications, 53(3), 164 168 .

[26] Nam, H., \& Han, B. (2016). Learning multi-domain convolutional neural networks for visual tracking. Paper presented at the Proceedings of the IEEE conference on computer vision and pattern recognition.

[27] Rahim, R., Afriliansyah, T., Winata, H., Nofriansyah, D., \& Aryza, S (2018). Research of Face Recognition with Fisher Linear Discriminant. Paper presented at the IOP Conference Series: Materials Science and Engineering.

[28] Ren, S., He, K., Girshick, R., \& Sun, J. (2015). Faster r-cnn: Towards real-time object detection with region proposal networks. Paper presented at the Advances in neural information processing systems.

[29] Sermanet, P., Eigen, D., Zhang, X., Mathieu, M., Fergus, R., \& LeCun, Y. (2013). Overfeat: Integrated recognition, localisation and detection using convolutional networks. arXiv preprint arXiv:1312.6229.

[30] Sun, Y., \& Yu, J. (2017). Facial expression recognition by fusing Gabor and Local Binary Pattern features. Paper presented at the International Conference on Multimedia Modeling.

[31] Taloba, A. I., Sewisy, A. A., \& Dawood, Y. A. (2018). Accuracy Enhancement Scaling Factor of Viola-Jones Using Genetic Algorithms. Paper presented at the 2018 14th International Computer Engineering Conference (ICENCO).

[32] Triantafyllidou, D., Nousi, P., \& Tefas, A. (2018). Fast deep convolutional face detection in the wild exploiting hard sample mining. Big data research, 11, 65-76.

[33] Uijlings, J. R., Van De Sande, K. E., Gevers, T., \& Smeulders, A. W. (2013). Selective search for object recognition. International journal of computer vision, 104(2), 154-171.

[34] Valueva, M. V., Nagornov, N., Lyakhov, P. A., Valuev, G. V., \& Chervyakov, N. I. (2020). Application of the residue number system to reduce hardware costs of the convolutional neural network implementation. Mathematics and Computers in Simulation, 177, 232 243.

[35] Viola, P., \& Jones, M. J. (2004). Robust real-time face detection. International journal of computer vision, 57(2), 137-154.

[36] Wan, S., Chen, Z., Zhang, T., Zhang, B., \& Wong, K.-k. (2016) Bootstrapping face detection with hard negative examples. arXiv preprint arXiv:1608.02236.

[37] Yang, P., \& Yang, G. (2016). Feature extraction using dual-tree complex wavelet transform and gray level co-occurrence matrix. Neurocomputing, 197, 212-220.

[38] Zhang, S., Zhu, X., Lei, Z., Wang, X., Shi, H., \& Li, S. Z. (2018). Detecting face with densely connected face proposal network Neurocomputing, 284, 119-127.

[39] Zitnick, C. L., \& Dollár, P. (2014). Edge boxes: Locating object proposals from edges. Paper presented at the European conference on computer vision.

[40] Bernstein B.(2017). Principal Component Analysis, Lecture slides, available online: //davidrosenberg.github.io/mlcourse/Archive/2017/Lectures/13-PCASlides.pdf [accessed on 19 January, 2021]. 